\title{
ENDOSCOPIC TREATMENT OF MULTIPLE PANCREATIC ABSCESSES CASE REPORT AND REVIEW OF THE LITERATURE
}

\author{
Vlastimil Prochazka ${ }^{a}$, Saaba Al-Eryani ${ }^{\mathrm{a}}$, Miroslav Herman ${ }^{\mathrm{b}}$
}

\author{
a Department of Internal Medicine II, Faculty of Medicine and Dentistry, Palacky University, Olomouc, Czech Republic \\ ${ }^{b}$ Department of Radiology, Faculty of Medicine and Dentistry, Palacky University, Olomouc, Czech Republic \\ e-mail:vlastimil.prochazka@fnol.cz
}

Received: September 25, 2008; Accepted: November 5, 2008

Key words: Pancreatic abscesses/Endoscopic drainage/Acute pancreatitis.

AIMS: Pancreatic abscesses are treated surgically and the role of endotherapy is still to be established. We describe the case of successful endoscopic management of two pancreatic abscesses in a critically ill patient.

METHODS: A patient was admitted to the hospital for severe acute pancreatitis. One month later the patient developed pancreatic sepsis. CT scan showed two large abscesses. The first was bulging to the posterior wall of the stomach and another at the tail of the pancreas. Parenteral antibiotic therapy was administered. The clinical status of the patient rapidly deteriorated and the patient was unfit for surgical intervention. The endoscopic retrograde cholangiopancreatography was performed. The pancreatic duct communicated with the abscess at the tail of the pancreas.

The drainage of this abscess was done transpapillarily. Endoscopic cystogastrostomy was performed to treat the pancreatic abscess that bulged to the posterior gastric wall. A double nasocystic tube was placed for continuous lavage of the abscess. Pseudomonas aeruginosa was cultured and antibiotics were administered according to sensitivity tests. The clinical status returned gradually to normal. A follow-up CT scan 4 months later showed complete resolution of abscesses.

RESULT: The drainage of the abscesses was done by the means of endoscopic cystogastrostomy and transpapillary stent insertion. This was a minimally invasive intervention, by which we avoided surgical intervention that bears significant mortality and morbidity.

CONCLUSION: Endoscopic drainge of pancreatic abscesses may be the therapy of choice in such patients mainly because it does not prevent the chance of subsequent surgical intervention if needed.

\section{ABBREVIATIONS}

Pancreatic pseudocysts (PPs), computed tomography (CT), pancreatic abscesses (PABS).

\section{INTRODUCTION}

Pancreatic pseudocysts (PPs) are reported to occur after the course of acute pancreatitis in $5 \%-10 \%{ }^{1}$.

The resolution of acute sterile PPs occurred in 8-70\% of patients ${ }^{2}$. The spontaneous resolution of PPs was reported to be higher if occurred after acute pancreatitis ${ }^{3}$.

Pancreatic abscess arises as a consequence of acute pancreatitis, pancreatic trauma or secondary infection of PPs. They often occur 4 weeks or more after the onset of pancreatitis $^{4}$.

Over the last decades the endoscopic drainage of PPs has become the therapy of choice for sterile pseudocysts with higher success, lower complications and recurrence rates than surgical approach ${ }^{5,6}$.

Despite the recent advances in endoscopic technology, pancreatic abscesses (PABS) are still treated surgically and the role of endotherapy is still to be established. Currently there are only a few reported cases regarding the role of endoscopic treatment of PABS with non-uniform results ${ }^{7,8}$.

Here we describe the case of successful endoscopic management of two large PABS presented in 4 weeks after the episode of acute pancreatitis.

\section{MATERIAL AND METHODS}

A 70years old patient was admitted to the hospital for severe abdominal pain without fever and chills. On admission, the laboratory results revealed leucocytosis $29.10 \mathrm{G} / 1$ (normal range 4-10 G/1), raised serum amylase $45.42 \mu \mathrm{kat} / 1$ (normal range $0.47-1.67 \mu \mathrm{kat} / 1$ ) and lipase levels 14.5 (normal range $0.22-1.2 \mu \mathrm{kat} / 1$ ). The serum $\mathrm{C}$ reactive protein on admission was $3.6 \mathrm{mg} / 1$ (normal range 0-12 mg/l) and increased to $217 \mathrm{mg} / 1$ within 48 hours.

On admission, a computed topography scan (CT scan) showed enlargement of the pancreas with peripancreatic fluid collection and necrosis at the tail of the pancreas without pseudocysts formation (Fig. 1). After conservative treatment the patient's symptoms resolved and leucocyte count, serum amylase and lipase returned to normal range.

One month later the patient developed fever with leucocytosis (14.93 g/1), elevated serum CRP (356 mg/1) and 


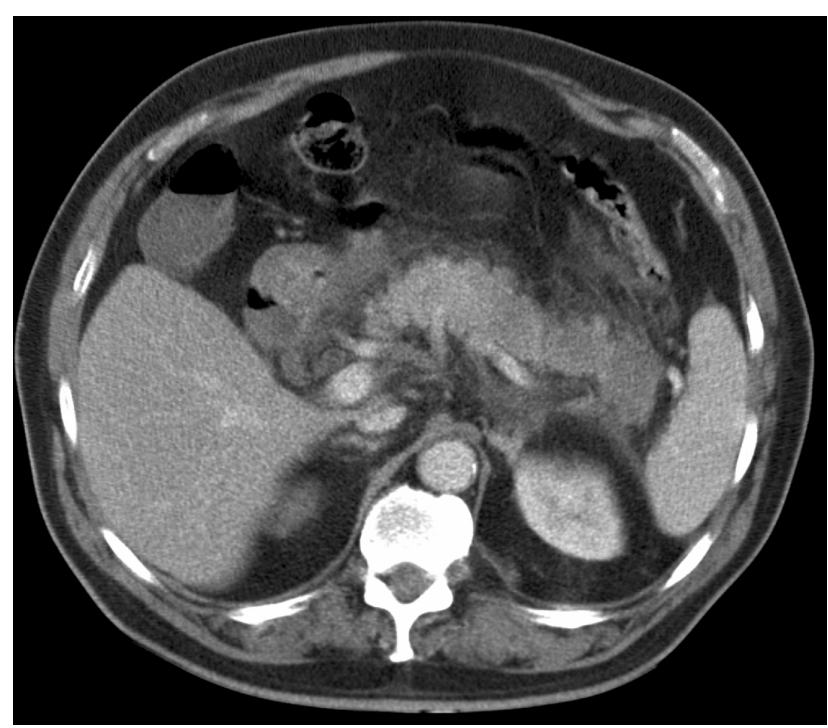

Fig. 1. CT scan on admission showing enlargement of the pancreatic gland, necrosis at the tail of the pancreas and peripancreatic fluid collections.

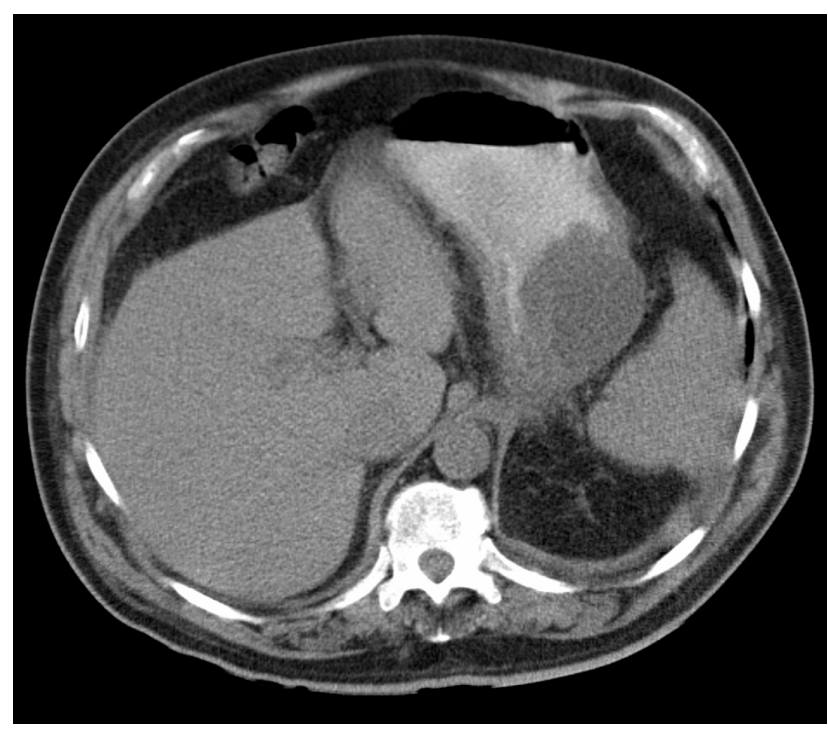

Fig. 3. CT scan one month after admission showing pseudocyst sized $(7.2 \times 5.7 \times 6.5 \mathrm{~cm})$ at the tail of the pancreas.

clinical signs of pancreatic sepsis. CT scan showed two large abscesses; the first was bulging to the posterior wall of the stomach (sized $9.5 \times 8.5 \times 8 \mathrm{~cm}$ ) and another (sized $7.2 \times 5.7 \times 6.5 \mathrm{~cm}$ ) at the tail of the pancreas (Fig. 2, 3).

Parenteral antibiotic therapy was administered and the patient was admitted to intensive care unit. The clinical status of the patient rapidly deteriorated and the patient was unfit for surgical intervention. Informed consent was obtained and endoscopic retrograde cholangiopancreatography (ERCP) was performed and revealed evidence of pancreatic duct stenosis at midbody of the pancreas with retrograde dilatation. The dilated pancreatic duct communicated with the abscess at the tail of the pancreas.

The drainage of this abscess was done transpapillarily

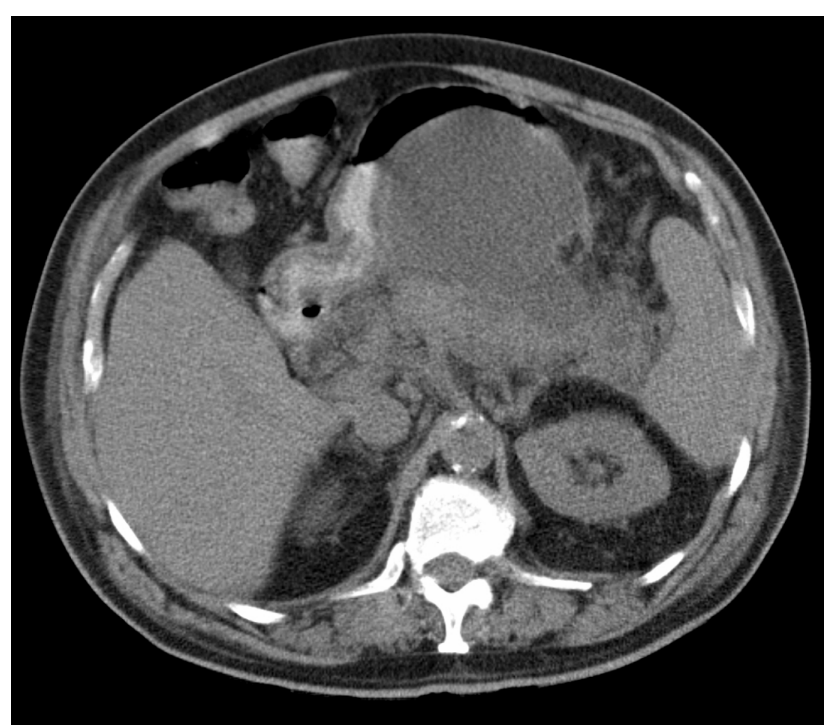

Fig. 2. CT scan one month after admission showing a cystic formation sized $(9.5 \times 8.5 \times 8 \mathrm{~cm})$ bulging to the posterior wall of the stomach.

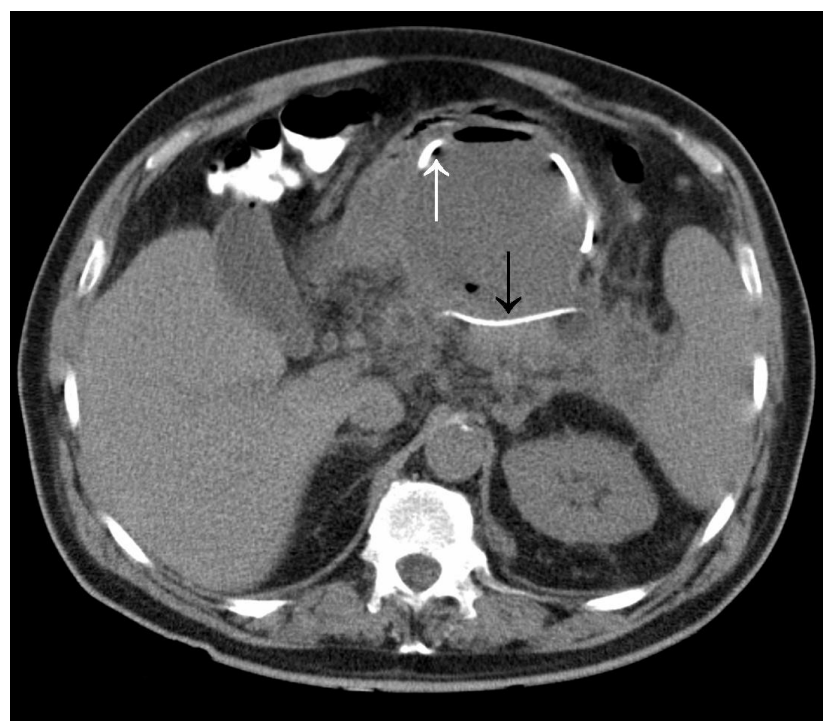

Fig. 4. CT scan after performing ERCP: nasocystic drain is seen inside the pancreatic abscess at the bursa omentalis (white arrow), transpapillary doublepigtail stent is seen inside the pseudocyst in pancreatic tail (black arrow).

by inserting a double pigtail stent (Zimmon-5 Fr - $22 \mathrm{~cm}$, Wilson Cooke Medical Inc) after pancreatic sphincter papillotomy and balloon dilatation of the pancreatic duct stenosis (Fig. 4, 5). A purulent material poured via the stent to the duodenum. The stent was repeatedly washed out with normal saline. Endoscopic cystogastrostomy was performed to treat the pancreatic abscess that bulged to the posterior gastric wall. After an incision was made at the maximal bulging of the abscess using a needle knife, a purulent fluid poured into the stomach. Subsequently a balloon dilatation of the incision site was done and a double nasocystic Liguory tube (Wilson Cooke Medical Inc) was placed for continuous lavage of the abscess (Fig. 4, 5). An aspirated sample of the purulent material was sent for 
microbiology and culture studies. Continuous lavage of the abscess took place for one week using Braunol ${ }^{\circledR}$ solution $7.5 \%$ (Iodopolyvidonum) at the dilution of $1: 40$. Two liters of diluted solution per day were used for the continuous lavage during the first three days, and $500 \mathrm{ml}$ per day for the remaining 4 days.

After the procedure, the clinical status of the patient gradually improved.

Pseudomonas aeruginosa was cultured from aspirated content of the abscesses and antibiotics were administered according to sensitivity tests. Temperature and the laboratory parameters returned gradually to normal. A follow up CT scan 6 weeks after the procedure showed only residuals after PABS and minimal changes in pancreatic parenchyma (Fig. 6). Another follow-up CT scan 4 months later showed complete resolution of PABS and disappearance of pancreatic heterogeneity (Fig. 7).

\section{RESULT}

The drainage of the abscesses was done by the means of endoscopic cystogastrostomy and transpapillary stent insertion. This was a minimally invasive intervention, by which we avoided surgical intervention that bears significant mortality and morbidity.

\section{DISCUSSION}

Patients with PABS are usually treated surgically. The high mortality and morbidity associated with surgical intervention mainly in critically ill patients illustrates the need of less invasive means ${ }^{9}$. The PABS are also difficult to manage with percutaneous techniques because of their high recurrence rates (around $20 \%$ ) and infec-
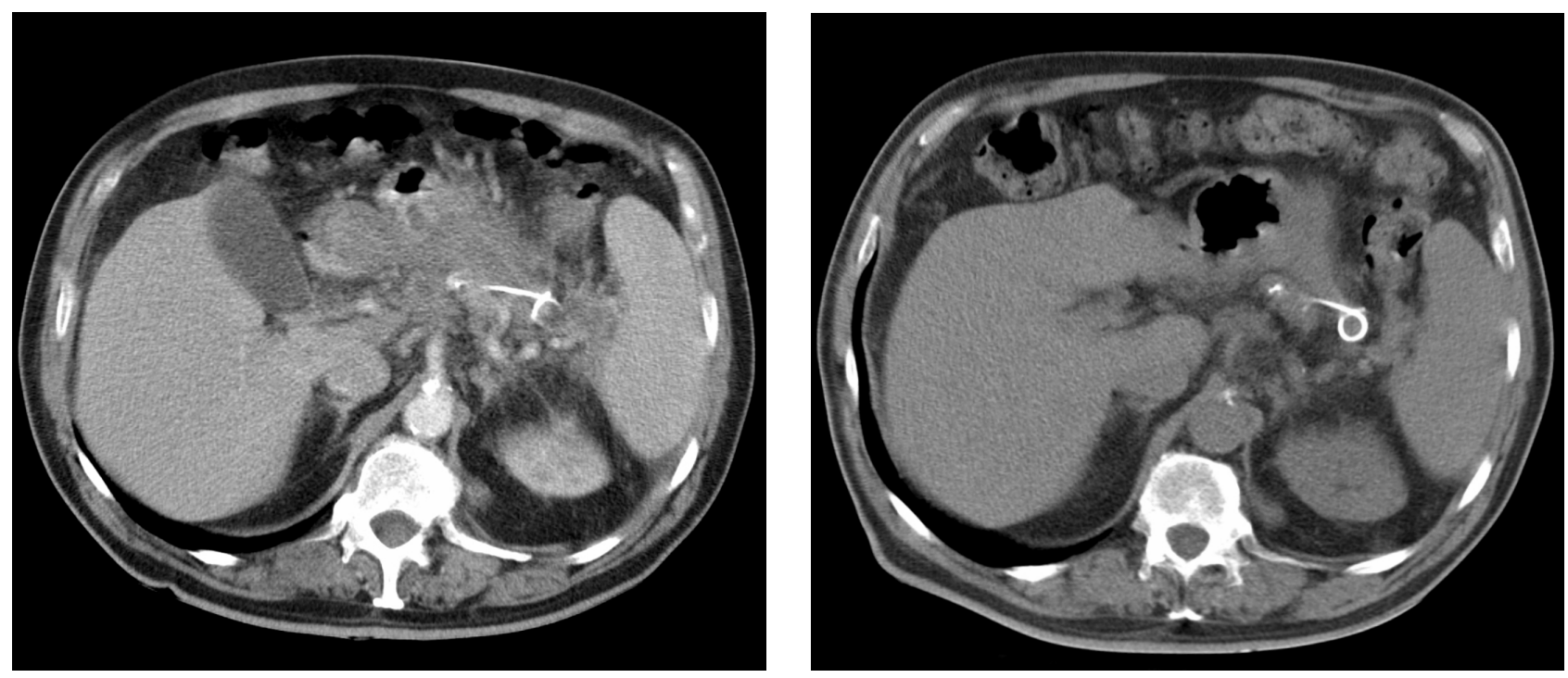

Fig. 6. Follow up CT scan after 6 weeks showing significant decrease of the size of both pancreatic abscesses with minimal changes of pancreatic parenchyma.

Fig. 7. Follow up CT scan after 4 months latter showing

tious complications that occurred in as many as $50 \%$ of patients ${ }^{10}$.

Although endoscopic therapy is well established for sterile PPs, the experiences with endoscopic drainage of PABS are very limited.

However, only very few reported cases are available regarding the use of endoscopic drainage in the treatment of PABS. The complete resolution observed in some of these studies was encouraging ${ }^{11}$. complete resolution of both pancreatic abscesses. The pigtail stent inserted through the pancreatic duct is still seen.

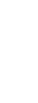


Our patient had two large PABS, one was communicating with the main pancreatic duct and the other was bulging to the stomach. Successfully, we drained the former transpapillarily after pancreatic sphincter papillotomy and balloon dilatation of the pancreatic duct stenosis.

Cystogastrostomy and nasocystic drainage was established for continuous evacuation and lavage of the latter. The procedure was carried out 2 days after the patient developed signs of pancreatic sepsis that was not responding to antibiotic therapy. The purulent appearance of pseudocystic content, its culture as well as the clinical and laboratory improvement after the procedure confirmed the diagnosis of PABS.

\section{CONCLUSION}

Our case study demonstrates the safety and effectiveness of endoscopic drainage of pancreatic abscesses. We suggest that endoscopic therapy may be considered as the first line therapy of PABS because it does not prevent the chance of subsequent surgical intervention if needed. Further studies are needed to determine the definite role of endoscopic drainage in the treatment of PABS.

\section{REFERENCES}

1. Ihse I, Andersson R. Acute pancreatic pseudocysts in Sweden. In: Bradley EL III ed. acute pancreatitis. Diagnosis and therapy. New York: Raven Press, 1994: 227-231.

2. McConnell DB, Gregory JR, Sasaki TM, Vetto RM: Pancreatic pseudocyst. Am J Surg 1982; 143:599- 601.

3. Warshaw AL, Rattner DW: Timing of surgical drainage for pancreatic pseudocyst. Clinical and chemical criteria. Ann Surg 1985; 202:720-4.

4. Frey C, Reber HA. Clinically based classification system for acute pancreatitis. Pancreas. 1993; 8:738-40.

5. Smits ME, Rauws EAJ, Tytgat GNJ, Huibregtse K: The efficacy of endoscopic treatment of pancreatic pseudocysts. Gastrointest Endosc 1995;42: 202-207.

6. Vitas GJ, Sarr MG: Selected management of pancreatic pseudocysts: operative versus expectant management. Surgery 1992; 111:123-30.

7. Fuchs M, Reimann FM, Gaebel C, Ludwig D, Stange E F: Treatment of Infected Pancreatic Pseudocysts by Endoscopic Ultrasonography-Guided Cystogastrostomy, Endoscopy 2000; 32: 654-657.

8. Shinozuka N, Okada K, Torii T, Hirooka E, Tabuchi S, Aikawa K et al.: Endoscopic pancreatic duct drainage and stenting for acute pancreatitis and pancreatic cyst and abscess. J Hepatobiliary Pancreat Surg. 2007;14:569-74.

9. Soliani P, Franzini C, Ziegler S, Del Rio P, Dell'Abate P, Piccolo D, et al.: Pancreatic Pseudocysts Following Acute Pancreatitis: Risk Factors Influencing Therapeutic Outcomes. Journal of Pancreas, 2004; 5:338-347.

10. vanSonnenberg E, Wittich GR, Casola G, Brannigan TC, Karnel F, Stabile BE et al.: Percutaneous drainage of infected and noninfected pancreatic pseudocysts: experience in 101 cases. Radiology. 1989; 170: 757-761.

11. Seewald S, Groth S, Omar S, Imazu H, Seitz U, de Weerth A et al.: Aggressive endoscopic therapy for pancreatic necrosis and pancreatic abscess: a new safe and effective treatment algorithm: Gastrointest Endosc. 2005; 62:92-100. 